\title{
Development of high damage threshold laser-machined apodizers and gain filters for laser applications
}

\author{
Patrick Rambo, Jens Schwarz, Mark Kimmel, and John L. Porter \\ Sandia National Laboratories, PO Box 5800, MS 1197, Albuquerque, NM 87185, USA \\ (Received 30 April 2016; revised 6 July 2016; accepted 5 August 2016)
}

\begin{abstract}
We have developed high damage threshold filters to modify the spatial profile of a high energy laser beam. The filters are formed by laser ablation of a transmissive window. The ablation sites constitute scattering centers which can be filtered in a subsequent spatial filter. By creating the filters in dielectric materials, we see an increased laser-induced damage threshold from previous filters created using 'metal on glass' lithography.
\end{abstract}

Keywords: apodization; laser beam shaping; laser micro-machining; spatial filtering

\section{Introduction}

Many laser applications, including high energy laser development, call for the generation of a high-order superGaussian ('flat top') beam profile. For high energy laser systems, where flat-top beams are desired to optimize energy extraction while minimizing spatial clipping ${ }^{[1]}$, certain common methods do not apply. Refractive beam shapers that use custom aspheric lenses are not used due to undesired potential ghost reflections and chromatic aberrations. Similarly, beam integrators (like relay imaged lenslets) and diffractive optics have a poor depth of focus and coherent artifacts which make them undesirable in a laser chain (although they may be used at a single plane such as a target plane). Rather, to generate flat-top beams, most high energy lasers around the world rely upon apodization methods, which involve the spatial filtering of a modulated near-field beam. Such modulation can be in amplitude or phase and can be generated by reflective, transmissive or scattering techniques. Most of these large laser systems apodize the beam early in the laser chain where the beam fluence is low, often using precise serrated-edge apodizers that are fabricated via 'metal on glass' lithography techniques ${ }^{[2]}$ or via laser cutting of thick foils. The 'metal on glass' method can be extended via continuous material deposition or a dithered binary deposition $^{[3]}$. Using these dithered or continuous 'metal on glass' methods, amplitude filters can be created and placed in the same low-fluence region to allow the compensation of gain profile nonuniformities in the main amplifiers ${ }^{[3-7]}$.

Correspondence to: P. Rambo, Sandia National Laboratories, PO Box 5800, MS 1197, Albuquerque, NM 87185, USA. Email: prambo@sandia.gov
However, there are situations where such filters and apodizers are desired in a higher fluence region of the beam. Such is the case in some high energy petawatt lasers which employ optical parametric chirped pulse amplification (OPCPA) for a first stage amplifier and traditional $\mathrm{Nd}$ :Phosphate Glass for subsequent amplifiers ${ }^{[6,8]}$. Rather than sending the OPCPA output directly into subsequent $\mathrm{Nd}$ :Phosphate Glass amplifiers, it is advisable to apodize and/or amplitude filter after the nonlinear gain process, allowing one to have better defined parameters at that position. Unfortunately, we have empirically observed 'metal on glass' apodizers placed in the chirped output of an OPCPA system damage at a modest $0.2 \mathrm{~J} \mathrm{~cm}^{-2}$ or less average fluence. One could attempt to optimize the metal material choice for higher damage threshold but the resulting damage threshold would still be inferior to dielectric materials.

The sum of these experiences has prompted interest in high damage threshold apodizers made from dielectric materials. One commercial source of apodizers (Continuum lasers) uses an anti-reflection coated window with a sandblasted periphery (in the style of Ref. [9]). The sandblasted area, with individual sites around $10 \mu \mathrm{m}$ in size, acts as a scattering region as opposed to the reflective region in a 'metal on glass' apodizer. As with a reflective or absorptive region, the scattering region can subsequently be removed from the beam at the pinhole of a spatial filter. These sandblasted optics have performed well with respect to damage threshold but tend to have a fairly gentle roll-off at the edges of the flat top, conforming to a sixth- to eighth-order super-Gaussian. Higher-order super-Gaussian profiles require better relay imaging in the amplifiers (due to diffractive propagation issues) but allow better energy extraction since the lower-order 
super-Gaussian profiles must use a smaller diameter beam to avoid clipping in the wings of the spatial profile. One could attempt fabricating a dielectric mirror with the appropriate reflection profile ${ }^{[10]}$ (sometimes called a gradient or variable reflectivity mirror). Other methods to shape beams have recently been demonstrated using static phase plates ${ }^{[11]}$ and polarization rotation in patterned liquid crystals ${ }^{[12]}$, both with fairly high damage thresholds. Exciting new active approaches are also being employed via spatial light modulators (SLM) with full phase and amplitude adjustment in the beam shaping ${ }^{[13,14]}$, although laser damage thresholds are still modest due to the SLM material.

While apodizers can be effectively used early in a laser amplifier chain, gain filters have a subtlety that requires further analysis. As mentioned, pre-compensation of gain profile nonuniformities has been done via several methods in positions in the chain where fluences are low. However, previous reporting has looked only at the spatial compensation. Gain saturation also leads to temporal pulse-shape distortions (PSD) ${ }^{[15]}$, which can also be pre-compensated with various methods ${ }^{[16-18]}$. Thus, pre-compensation of a nonuniform spatial gain profile at a single point early in an amplifier chain can have some degree of spatial variation in the PSD throughout a laser amplifier chain. A better answer is to stagewise apply gain filtering. As the fluences typically increase progressively down a laser amplifier chain, such a stagewise pre-compensation will require high laser damage threshold materials, making more precise and modern methods like the SLM approach less viable.

As a relatively simple cost effective and robust alternative, we propose creating a precise dither patterned structure using laser machining on anti-reflection coated window substrates. The machined region will act as a scatterer in much the same way as the sandblasted apodizers but the control of shape and edge roll-off can be much more precise due to the controlled fabrication technique. The idea has been demonstrated previously ${ }^{[19-21]}$ but standard nanosecond-scale laser machining runs the risk of inducing material stress birefringence due to the large heat affected zone. The use of a short pulse laser (less than $20 \mathrm{ps)}$ is ideal to mitigate this stress birefringence issue. We will demonstrate the concept using a pick-off of an available $10 \mathrm{~Hz}$ OPCPA operating at $500 \mathrm{fs}$ and $\leqslant 10 \mathrm{~mJ}$ to perform the fabrication.

\section{Beam-shaping motivation}

The proposed beam shaping is for a $\mathrm{Nd}$ :Phosphate Glass rod amplifier system acting as the front end of a larger slab amplifier system. The system is designed to amplify a beam with a high-order super-Gaussian spatial profile using cascaded double-pass flash-lamp pumped rod amplifiers of sizes 16, 25, 45 and $64 \mathrm{~mm}$ diameter (all $L=235 \mathrm{~mm}$ length).

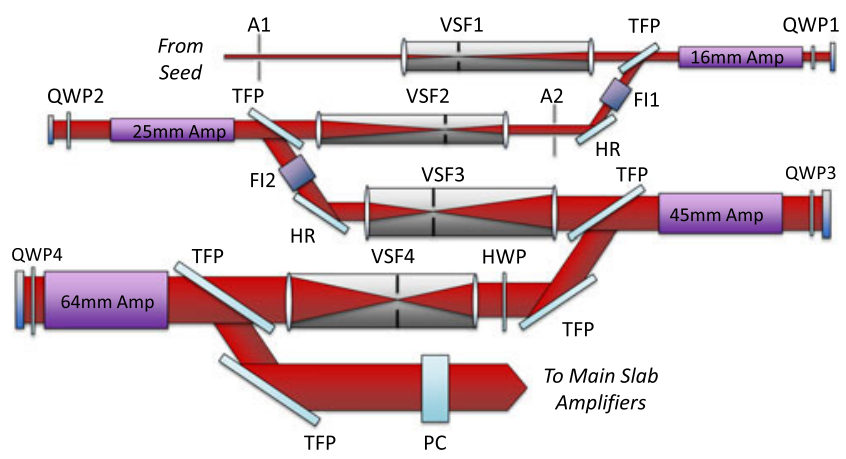

Figure 1. Notional rod amplifier layout. A1 and A2 are apodizer and gain filter planes. VSF1, VSF2, VSF3 and VSF4 are vacuum spatial filters with $2.5 \mathrm{x}, 2 \mathrm{x}, 1.875 \mathrm{x}$ and $1.4 \mathrm{x}$ magnification respectively. TFPs are thin film polarizers. HRs are high reflector mirrors. QWP1, QWP2, QWP3 and QWP4 are zero-order quarter-wave plates while HWP is a zero-order halfwave plate. FI1 and FI2 are $12 \mathrm{~mm}$ and $25 \mathrm{~mm}$ Faraday isolators (which include a half-wave plate). PC is the final $75 \mathrm{~mm}$ clear aperture Pockels cell. Apodizer A2 defines an object plane for relay imaging, with image planes at the entrance to FI2 and the entrance to VSF4.

All amplifiers may not be used at the same time. Each amplifier is separated by magnifying vacuum spatial filters (shown notionally in Figure 1) ${ }^{[22]}$. Note that the quarterwave plate placements are ideally on the input side of the rods (with the circular polarization lowering the nonlinear refractive index and the resulting B-integral in the rods ${ }^{[23]}$ ) but proximity of the rod amplifiers to the nearby polarizers generally prevents this. As such, the quarter-wave plates are placed between the rods and end mirrors. The designed full-width at half-maximum (FWHM) beam size is 10, 20, 37.5 and $52.8 \mathrm{~mm}$ in each amplifier respectively, targeting a maximum fill factor (which we consider to be ratio of the FWHM to the rod diameter) of $80 \%$ to $83 \%$ for efficient energy extraction with sufficient clear aperture for alignment. The double-pass gain after each amplifier was measured in two manners: first by simply measuring the output and input energies and second by measuring the output and input nearfield beam profiles (using calibrated neutral density filters as necessary). In doing this, the output fluences were kept to a only few percent of the $5.6 \mathrm{~J} \mathrm{~cm}^{-2}$ saturation fluence for the Schott APG-1 Nd:Phosphate glass used in the system. This avoids saturation effects and measures the double-pass small signal gain. Two-dimensional (2D) fits of the doublepass small signal gain $G_{0}(r)$ as a function of radius $r$ were done using an exponential function decaying inward from the perimeter (following a Beers Law absorption of the pump light). The 2D fit allows one to account for gain asymmetry that may be associated with beam centration issues in the rod. $G_{0}(r)$ for each rod was only measured to the seed beam radius, which is $\sim 80 \%$ of the clear aperture.

As seen in a sample $G_{0}(r)$ in Figure 2, the fits of the gain profiles typically show $>2.5 x$ variation on the gain from the central axis to the edge of the beam, which is largely driven by the rod doping levels, flash-lamp pump energies 
(a)
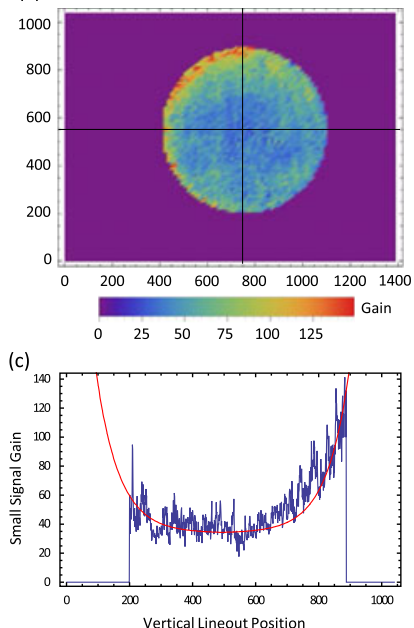

(b)

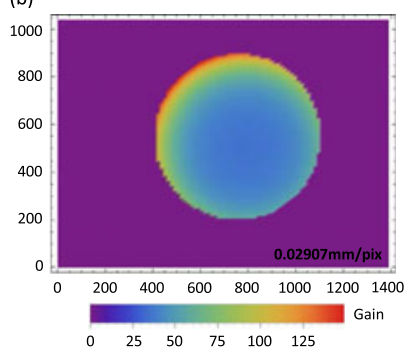

(d)

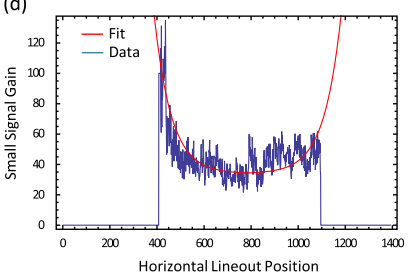

Figure 2. Fits for double-pass small signal gain $G_{0}(r)$ for a $25 \mathrm{~mm}$ rod amplifier. (a) Measured 2D gain profile $G_{0}(r)$ at $64 \mathrm{~mm}$ near-field equivalent plane. (b) 2D fit to measured data using an asymmetrically placed exponentially decaying function. (c) Vertical lineout and fit. (d) Horizontal lineout and fit.

and amplifier timing. The compounding of the rod gain variations (in conjunction with the subsequent main slab amplifiers) can lead to higher fluences at the beam perimeter, causing a concern of damage. In addition, temporal PSD comes into play, exacerbating the risk of damage.

\section{Designing and verifying a dither pattern}

With the radial gain profiles known, a continuous gain filter is designed to pre-compensate the gain of the various rods (see Figure 1). Assuming a flat-top input beam, the product of the designed gain filter transmission and the measured cumulative gain should yield a flat-top beam profile. The desired pre-compensating numerical grayscale filter in 2 dimensions is then converted to an array of dots using Floyd-Steinberg error diffusion ${ }^{[3]}$, with the black dots in the pattern representing opaque/scattering regions and the white regions being fully transmissive. Note that, in largely transmissive areas (i.e., center), the error diffusion pattern tends to generate a few undesirable dots. As such, a small masking disk layer is overlaid in the center to eliminate these errant points.

Pixel sizes in the dither pattern should be chosen to balance effective spatial filtering with processing manageability. In this sense, we consider a serrated-edge apodizer design based upon the concepts outlined in Auerbach and Karpenko $^{[2]}$. For an apodizer which is subsequently filtered by a spatial filter with an input lens focal length of $f$ and a pinhole radius of $a$, the allowed serration size $L$ (i.e., tooth width) is:

$$
2 \cdot a=(f \cdot \lambda) / L,
$$
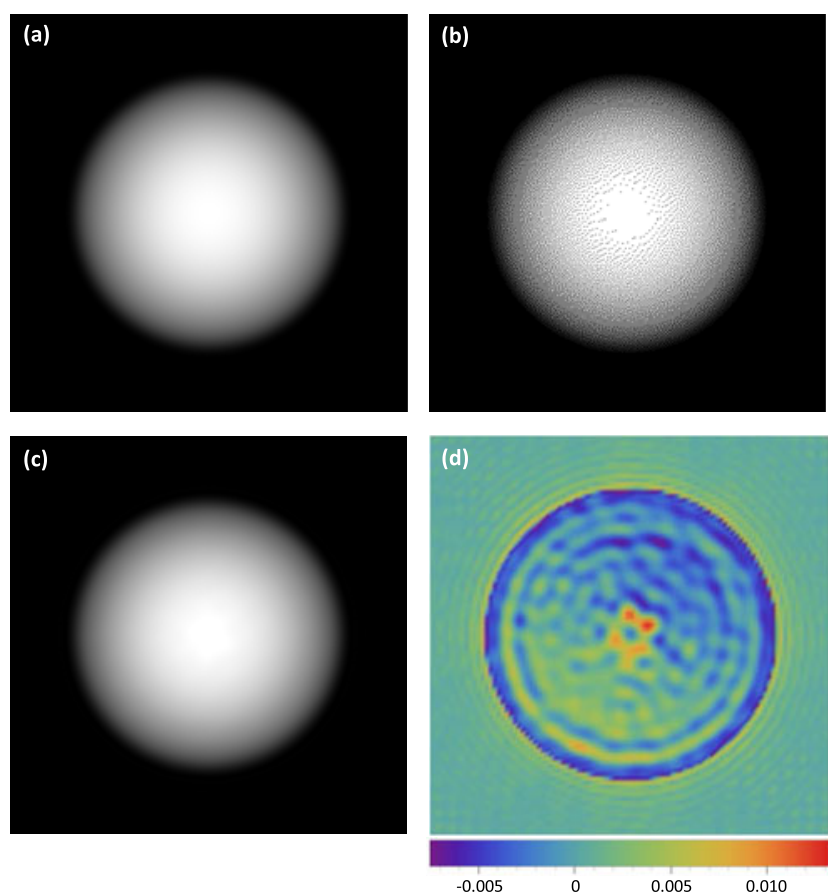

Figure 3. Simulated spatial filtering of a dither pattern. (a) 2D continuous grayscale of ideal radial gain filter. (b) $2 \mathrm{D}$ error diffusion dither pattern (scale: $16 \mathrm{~mm} \times 16 \mathrm{~mm}$; pixel size: $40 \mu \mathrm{m} \times 40 \mu \mathrm{m}$ ). (c) Simulation of spatially filtered dither pattern. (d) False color view of the continuous filter image (a) subtracted from the dithered filtered image (c) with the scale shown relative to the peak of 1 in (a).

where $\lambda$ is the laser wavelength in use. If using $\lambda=1054 \mathrm{~nm}$ with a lens of $f=763 \mathrm{~mm}$ and a pinhole of $2 \cdot a=$ $1.75 \mathrm{~mm}$, the serrated tooth width should be $460 \mu \mathrm{m}$ or less. Assuming the serrations were made of pixels, one would want at least 10 pixels per tooth to sufficiently resolve features, meaning dither patterned pixels in this case should be $46 \mu \mathrm{m}$ or less. As such, we have assumed an upper end of $40 \mu \mathrm{m}$ pixel size, which reduces overall array sizes (for a given spatial dimension) while demonstrating effective spatially filtered performance. To check this performance, we consider an infinite flat-top beam going through the dithered gain filter (see Figure 3(b)) that is based upon the ideal continuous filter (see Figure 3(a)). Thus, a simple 2D fast Fourier transform (FFT) of the dither pattern can be used to simulate the far-field beam profile at the pinhole plane of a spatial filter. After calibrating this Fourier plane to the appropriate spatial frequency observed experimentally at one of the actual pinhole planes, a circular low-pass transmission filter can be multiplied with the 2D-FFT of the beam profile. This now numerically represents the transmission of the actual pinhole used in one of the spatial filters. A subsequent inverse 2D-FFT represents the output near-field beam profile, showing the quality of the spatially filtered dither pattern (see Figure 3(c)). As one can see in Figure 3(d) (which shows the difference in the ideal and spatially filtered dithered filters), the filtered dithered 
near-field beam profile does a good job of reproducing the original continuous gain filter, with some low modulation structure observable from the dither points at the level of a few percent. If the filtered beam were to show too much structure, one could reduce the pinhole size or reduce the pixel size used in the dither pattern in order to create a better fidelity of the filtered dither pattern to the original continuous gain filter, although both changes have other ramifications (such as beam profile modification and increased fabrication time, respectively).

\section{Fabricating a dither patterned filter}

With the dither pattern created, the fabrication of a dithered filter can be performed with laser micro-machining of an anti-reflection coated window. This laser micro-machining is done using pick-off light from the Z-Petawatt front-end OPCPA system ${ }^{[8,24]}$ operating at $10 \mathrm{~Hz}, 500 \mathrm{fs}, \leqslant 10 \mathrm{~mJ}$ and $1054 \mathrm{~nm}$ with a $\phi 1 \mathrm{~cm}$ beam. The use of an ultrashort pulse laser system helps to minimize the heat-affected zone near the laser ablated region, which should in turn mitigate any induced stress birefringence from the micro-machining. The machining system (see Figure 4) utilizes infrastructure developed for laser-damage testing, which employs several actuator control points. These include $x-y-z$ translation of the target optic, a half-wave plate (which together with a polarizer allows attenuation of the input energy at $1054 \mathrm{~nm}$ ), and a shutter (used to block the beam during translation). Using inputs from a tab-delimited ASCII file, custom LabView software drives the $x-y-z$ stages to a fixed position, adjusts the energy via the half-wave plate and then opens the shutter for a fixed number of shots. The shutter then closes and the target is moved to the next position where the process continues until all positions in the file have been exposed. Two low energy $(0.1-0.2 \mathrm{~mJ})$ pulses per site appear to create fairly round spots of $\sim 40 \mu \mathrm{m}$ diameter. Higher energies tend to yield noncircular holes and are irreproducible in size. In addition, higher energies may be subject to significant nonlinear effects in the ambient air. At $40 \mu \mathrm{m}$ diameter and $0.2 \mathrm{~mJ}$ in $500 \mathrm{fs}$, the intensity is $3.2 \times 10^{14} \mathrm{~W} \mathrm{~cm}^{-2}$, which is just below the intensity clamping threshold in air of $3.5 \times 10^{14} \mathrm{~W} \mathrm{~cm}^{-2}$ observed for this system ${ }^{[25]}$. The laserdamage test system used for this laser machining can be used with the target in vacuum to avoid these atmospheric effects but prior damage testing has indicated that damage sites created in vacuum show signs of debris around the burn spot (which would be undesirable in a machined gain filter) ${ }^{[26]}$. Inert gas flow near the ablation site during fabrication, which is somewhat common in laser-cutting work, was not tried here. The use of such inert (noble) gases, usually at pressures elevated above ambient conditions for laser cutting, in the vicinity of an ultrashort pulse focal region would require additional thought to account for the possible impact of selffocusing. Gas type would also become relevant as some

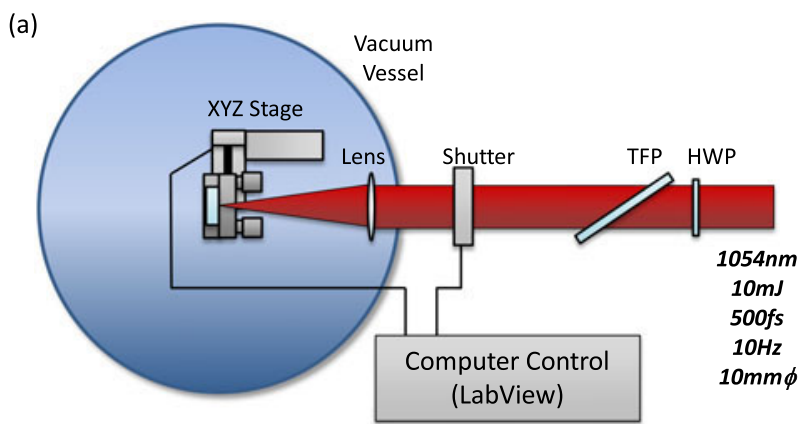

(b)

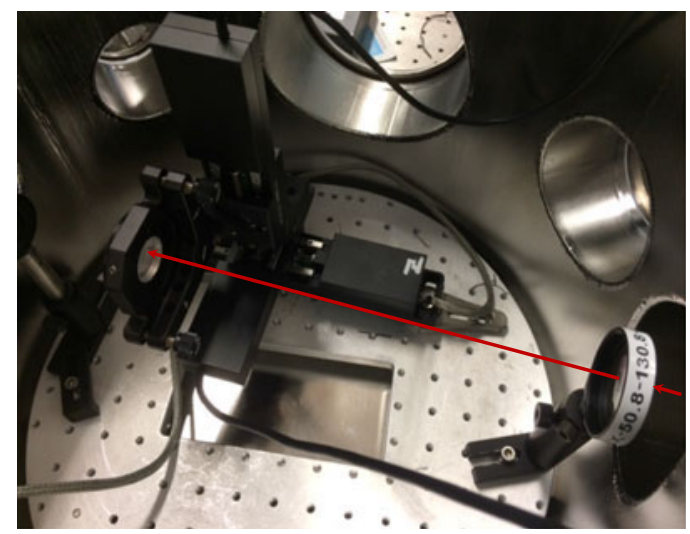

Figure 4. Laser-machining setup. (a) Schematic. (b) Photograph.

noble gases have a lower nonlinear refractive index than air and some have a significantly higher nonlinear refractive index.

In addition to spot size selection issues, using a round laser burn to approximate a square pixel (with the circular burn spot inscribed within the square pixel) results in an areal underfill of $21.5 \%$, meaning an opaque pixel in the ideal dither pattern still transmits $21.5 \%$ at the real pixel corners. In general, the effect degrades the contrast depth on the transmitted beam that can be achieved with a simple laser-machined gain filter. This shortcoming can be dealt with by cascading, using a unique gain filter at relay planes prior to different spatial filters. Another option would be to generate square laser focal spots to be used in the machining process.

Fabrication time is a practical issue in laser machining. Ideally, pixel sizes would be $10-20 \mu \mathrm{m}$ for better spatially filtered beam uniformity but the smaller pixels result in unreasonable fabrication times. Similarly, the gain filter dimensions also drive fabrication time, with larger dimensions requiring longer times. As such, a master array of $16 \mathrm{~mm} \times 16 \mathrm{~mm}$ was chosen, which for $40 \mu \mathrm{m}$ square pixels yields an array of $400 \times 400$ pixels (or $1.6 \times 10^{5}$ individual points). To reduce the number of written pixels, the gain filter is taken out to a radius slightly past where the nominal fully opaque region starts. At the $40 \mu \mathrm{m}$ pixel size, the larger gain filter shown in Figure 5(a) has $2.4 \times 10^{4}$ individual burn sites, which is a significant reduction to $15 \%$ of the 


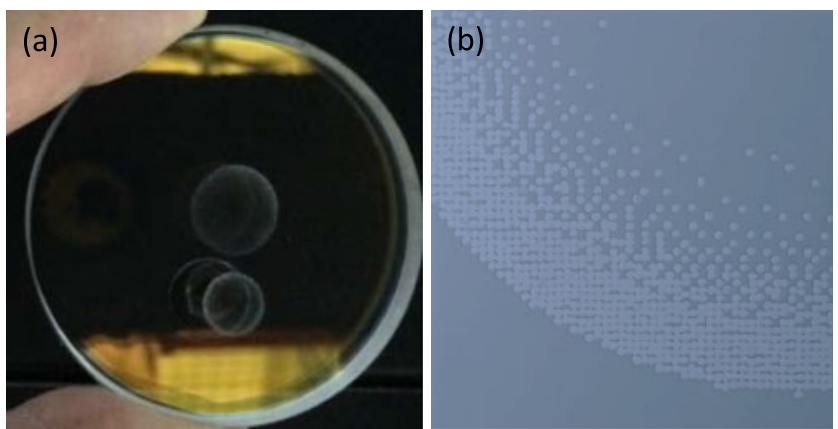

Figure 5. Sample laser-machined filters. (a) Sample Gain Filter (2 different sized samples on a 2 inch optic). (b) Sample Apodizer (5x microscope view).

available sites. The laser repetition rate, drive software, low velocity/cost stages and shutter used here all slow down the integrated fabrication time, with the larger pattern of 24000 points taking about $14 \mathrm{~h}$ to create. While time consuming, it is useful to perform the development in-house. Later pieces may be outsourced to commercial laser micro-machining businesses with faster integrated fabrication times.

There are a few techniques that can be used to reduce the fabrication time. Obviously, changes to the software or mechanical stages can improve matters. Acceleration and deceleration of the motorized stages takes time so one could consider rapid shuttering in conjunction with steady translation at a uniform speed. A very practical option would be to nest arrays with different pixel sizes similar in manner to half-tone printing processes. In the micromachining context, a layer of the desired filter is created for the smallest feature size at low pixel density. As the pixel density increases, a super-pixel formed by a $2 \times 2$ or $3 \times 3$ array of smaller pixels could be removed from the existing layer and placed into a new layer with a corresponding larger pixel size. As the pixel size grows and the layer changes, the focal position of the machining lens would be shifted to create a larger laser spot size and/or the laser energy would be increased to create a larger ablated region. The impact of this half-toning method is small unless the machined part has higher pixel densities over larger portions of the part.

\section{Laser-machined filter performance}

The filter was first assessed by comparing the filter performance to its design. To do this, a filter was placed at the A2 position in Figure 1 and the filter was illuminated by a uniform flat-top $\mathrm{CW}$ beam. After spatial filtering, the equivalent near-field diagnostic plane after the $64 \mathrm{~mm}$ rod amplifier showed the beam in Figure 6. The lineouts of the resulting near-field centroid did not initially compare well with the design. Only when accounting for the areal underfill issue (i.e., round burns approximating a square pixel) did the design and measured performance agree.

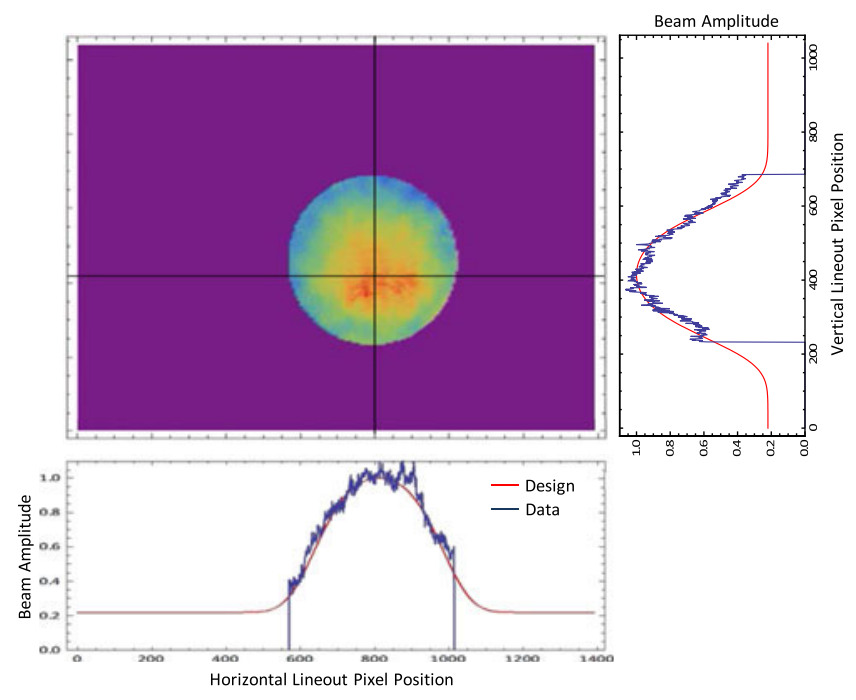

Figure 6. Sample performance of laser-machined gain filter. The false color image shows the spatially filtered near-field of a gain filter illuminated by a flat-top CW beam. Normalized horizontal and vertical lineouts (positioned on the respective centroids within the viewing aperture) are compared to the specified design (accounting for the area underfill issue mentioned in the text).

Note that, as indicated in Figure 1, the apodizers proceed through multiple crossed polarizers in the laser chain. As such, any local stress birefringence beyond the damage site from the laser-machining process simply enlarges the effective dither pattern pixel size.

Further testing of the gain filters occurred on beams that were amplified with the rod amplifier chain. To provide a baseline, a pulsed spatially filtered Gaussian profile seed beam ( $\leqslant 10 \mathrm{~mJ}, 1054 \mathrm{~nm}$ (narrowband), $2 \mathrm{~ns}$ ) was amplified in the rod chain using the 16, 45 and $64 \mathrm{~mm}$ diameter rods. The resulting near-field is shown in Figure 7(a). That shot showed 20000x gain and boosted a $0.26 \mathrm{~mJ}$ seed to $5.2 \mathrm{~J}$. The radial gain nonuniformity leads to a higher intensity annulus at the beam edge, yielding a peak-to-average value of 3.60 and a standard deviation to average value of 0.46 within the beam profile. A histogram of the overall near-field image data shows two humps, with the left hump pertaining to the peak from the background and the right hump pertaining to the average signal value in the beam. The tail extending toward the higher values indicates high beam intensities within the beam profile.

Pertaining to the layout from Figure 1, a softer edged apodizer was applied at the initial object plane (A1) before any of the amps and a gain filter was applied at the next relay plane (A2) just after the $16 \mathrm{~mm}$ amp. The use of cascaded filters was to deal with the nonoptimal beam extinction at the beam profile edge associated with the areal underfill issue on the filters. Both of these filters were fabricated at Sandia using the laser-machining approach. These apodizers and gain filters were placed in a low- to mid-fluence part of the amplifier chain. While not explicitly 
(a)

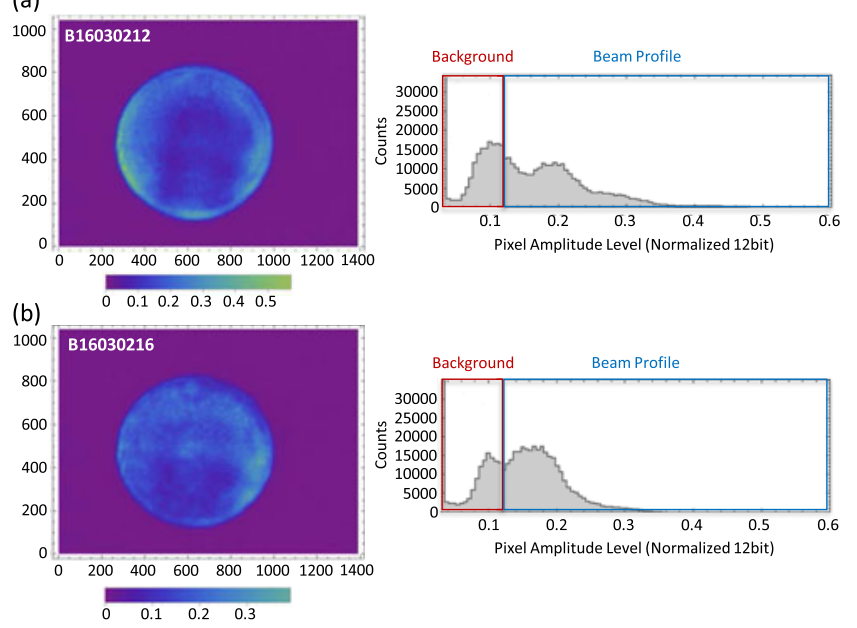

Figure 7. Sample performance of laser-machined gain filter on amplified shot. The false color images are 12-bit near-field beam profile data taken after the $64 \mathrm{~mm}$ rod. The histograms show the counts at a given intensity value over the whole near-field image. (a) Left: Near-field to beam without soft-edged apodizer and gain filter; right: Histogram of unfiltered near-field data. (b) Left: Near-field to beam with soft-edged apodizer and gain filter; right: Histogram of filtered near-field data.

damage tested (in part due to the heterogeneous nature of our filters), our laser-machined filters have been tested in moderate fluence parts of our chain (with average fluences up to $0.39 \mathrm{~J} \mathrm{~cm}^{-2}$ having been tested incident on the gain filter at the A2 position) without further damage. This is a higher operational fluence than metal-based apodizers that we have previously used, which tended to damage at less than $0.2 \mathrm{~J} \mathrm{~cm}^{-2}$ for our $2 \mathrm{~ns}$ pulsewidth at $1054 \mathrm{~nm}$. Using a similar seed energy value to the baseline case while going through the same active amplifiers, a filtered amplified shot showed 23500X gain and boosted a $0.20 \mathrm{~mJ}$ seed to $4.7 \mathrm{~J}$. As seen in Figure 7(b), the amplified beam shows a lower nonuniformity, yielding a peak-to-average value of 2.60 and a standard deviation to average value of 0.34 within the beam profile. The corresponding histogram still has two humps but the rightmost one is now narrower and lacks the higher value tail, indicating a more uniform beam profile. One should note that a static gain filter will only do a good job of precorrecting radial gain nonuniformity if the laser gain profiles in each rod are fairly consistent. Shy of measuring the radial gain profile of each rod on each shot, this consistency is reflected in the easily measured integrated gain of the rod amplifier chain on each shot. In our case, comparing similar configurations, the laser gain of the chain tends to be within a maximum $\pm 10 \%$ range of an average value.

\section{Conclusion}

Radial gain variations in rod amplifiers are non-negligible but can be compensated with the proper gain filters. In order to deal with such beam-shaping issues, we have demonstrated a new way to precisely fabricate high damage threshold apodizers and gain filters. The approach is convenient for most users of high power lasers because it allows such users to create said apodizers and filters themselves in a relatively simple setup. Performance of these filters is decent but not optimal, in part due to the poor contrast associated with imperfect spatial filling of the filter plane. This poor contrast can be addressed somewhat elaborately by using shaped (square) focal spots in the machining process. The latter square pixel approach could be dealt with easily via lithographic methods that tend to have fairly quick fabrication in comparison to the laser-damage system described here. However, to optimize the laser damage threshold advantage, a lithographic approach to gain filters or apodizers should involve etching a dielectric substrate rather than a metal top layer, which in turn would take longer than using lithography to fabricate a 'metal on glass' part. Regarding speed of fabrication, the laser-damage method of fabrication could be improved with a higher repetition rate system and rapid beam routing via a beam deflection approach.

The poor contrast associated with round pixels can also be addressed by using multiple cascaded filters (at different relay planes). The stagewise or cascaded compensation of gain variation in the rod amplifiers is uniquely allowed by the high laser-induced damage threshold of the dielectric gain filters and apodizers. Future testing will focus on these filter contrast issues and the damage threshold information.

\section{Acknowledgements}

Sandia is a multiprogramme laboratory operated by Sandia Corporation, a Lockheed Martin Company, for the U.S. Department of Energy's National Nuclear Security Administration under contract DE-AC04-94AL85000.

\section{References}

1. P. K. Rambo, I. C. Smith, J. L. Porter, M. J. Hurst, C. S. Speas, R. G. Adams, A. J. Garcia, E. Dawson, B. D. Thurston, C. Wakefield, J. W. Kellogg, M. J. Slattery, H. C. Ives, R. S. Broyles, J. A. Caird, A. C. Erlandson, J. E. Murray, W. C. Behrendt, N. D. Neilsen, and J. M. Narduzzi, Appl. Opt. 44, 2421 (2005).

2. J. M. Auerbach and V. P. Karpenko, Appl. Opt. 33, 3179 (1994).

3. C. Dorrer and J. D. Zuegel, J. Opt. Soc. Am. B 24, 1268 (2007).

4. B. M. VanWonterghem, J. R. Murray, J. H. Campbell, D. R. Speck, C. E. Barker, I. C. Smith, D. F. Browning, and W. C. Behrendt, Appl. Opt. 36, 4932 (1997).

5. M. J. Guardalben and L. J. Waxer, Proc. SPIE 7916, 79160G (2011).

6. N. Hopps, C. Danson, S. Duffield, D. Egan, S. Elsmere, M. Girling, E. Harvey, D. Hillier, M. Norman, S. Parker, P. Treadwell, D. Winter, and T. Bett, Appl. Opt. 52, 3597 (2013). 
7. Y. Peng, J. Wang, Z. Zhang, D. huang, W. Fan, and X. Li, Chin. Opt. Lett. 12, 041402 (2014).

8. J. Schwarz, P. Rambo, M. Geissel, M. Kimmel, E. Brambrink, B. Atherton, and J. Glassman, Opt. Commun. 281, 4984 (2008).

9. N. Rizvi, D. Rodkiss, and C. Danson, Rutherford Appleton Laboratory Annual Report RAL-87-041, 113 (1987).

10. S. De Silvestri, V. Magni, O. Svelto, and G. Valentini, IEEE J. Quantum Electron. 26, 1500 (1990).

11. C. Dorrer, Opt. Lett. 34, 2330 (2009).

12. C. Dorrer, S. K.-H. Wei, P. Leung, M. Vargas, K. Wegman, J. Boulé, Z. Zhao, K. L. Marshall, and S. H. Chen, Opt. Lett. 36, 4035 (2011).

13. S. W. Bahk, I. A. Begishev, and J. D. Zuegel, Opt. Commun. 333, 45 (2014).

14. S. Li, Y. Wang, Z. Lu, L. Ding, P. Du, Y. Chen, Z. Zheng, D. Ba, Y. Dong, H. Yuan, Z. Bai, Z. Liu, and C. Cui, Opt. Express 23, 681 (2015).

15. W. Koechner, Solid-State Laser Engineering 5th edn (Springer-Verlag, 1999).

16. J. Schwarz, P. Rambo, I. Smith, and J. Porter, Opt. Eng. 44, 094203 (2005).

17. D. N. Schimpf, C. Ruchert, D. Nodop, J. Limpert, A. Tnnermann, and F. Salin, Opt. Express 16, 17637 (2008).
18. L. Xu, L. Yu, Y. Chu, Z. Gan, and X. Liang, Appl. Opt. 54, 357 (2015).

19. L. M. Vinogradsky, V. A. Kargin, S. K. Sobolev, I. G. Zubarev, M. V. Pyatakhin, Y. V. Senatsky, A. V. Shelobolin, V. M. Mizin, and K.-I. Ueda, Proc. SPIE 3889, 849 (2000).

20. M. V. Pyatakhin and Yu V. Senatsky, J. Russ. Laser Res. 23, 332 (2002).

21. P. K. Rambo, J. Schwarz, M. Kimmel, B. W. Atherton, A. Bergstrom, and B. Flusche, in Conference on Lasers and Electro-Optics/Quantum Electronics and Laser Science Conference and Photonic Applications Systems Technologies (2008), paper CMO5.

22. J. T. Hunt, J. A. Glaze, W. W. Simmons, and P. A. Renard, Appl. Opt. 17, 2053 (1978).

23. W. Seka, J. Soures, O. Lewis, J. Bunkenburg, D. Brown, S. Jacobs, G. Mourou, and J. Zimmermann, Appl. Opt. 19, 409 (1980).

24. J. Schwarz, P. Rambo, M. Geissel, A. Edens, I. Smith, E. Brambrink, M. Kimmel, and B. Atherton, J. Phys.: Conf. Ser. 112, 032020 (2008).

25. J. Schwarz, P. Rambo, M. Kimmel, and B. Atherton, Opt. Express 20, 8791 (2012)

26. M. Kimmel, P. Rambo, R. Broyles, M. Geissel, J. Schwarz, J. Bellum, and B. Atherton, SPIE 7504, 75041G (2009). 